04

\title{
Исследование пристеночной плазмы токамака Глобус-М с помощью массива диверторных ленгмюровских зондов
}

\author{
(ㄱ Н.А. Хромов, ${ }^{1}$ Е.О. Векшина, ${ }^{2}$ В.К. Гусев, ${ }^{1}$ Н.В. Литуновский, ${ }^{3}$ М.И. Патров, ${ }^{1}$ Ю.В. Петров, ${ }^{1}$ Н.В. Сахаров ${ }^{1}$ \\ ${ }^{1}$ Физико-технический институт им. А.Ф. Иоффре РАН, \\ 194021 Санкт-Петербург, Россия \\ ${ }^{2}$ Санкт-Петербургский политехнический университет Петра Великого, \\ 194021 Санкт-Петербург, Россия \\ ${ }^{3}$ Научно-исследовательский институт электрофизической аппаратуры им. Д.В. Ефремова, \\ 196641 Санкт-Петербург, Россия \\ e-mail: Nikolay.Khromov@mail.ioffe.ru
}

Поступило в Редакцию 16 июля 2020 г.

В окончательной редакции 14 сентября 2020 г.

Принято к публикации 15 сентября 2020 г.

Массив из десяти встроенных диверторных зондов был установлен на нижний купол сферического токамака Глобус-М. С его помощью проведены измерения профилей плавающего потенциала, электронной температуры, ионного тока насыщения и плотности потока тепла на дивертор. Продемонстрировано резкое изменение плавающих потенциалов зондов, находящихся вблизи внешней ветви сепаратрисы, при развитии периферийной неустойчивости.

Ключевые слова: токамак, дивертор, пристеночная плазма, ленгмюровские зонды.

DOI: 10.21883/JTF.2021.03.50518.227-20

\section{Введение}

Одной из самых серьезных проблем для термоядерных установок с магнитным удержанием плазмы является высокая тепловая нагрузка компонентов, обращенных к плазме. Особенно это касается сферических токамаков, так как в них потоки тепла из плазмы попадают на сравнительно небольшую площадь [1]. Поэтому для подобных установок задача измерения параметров пристеночной плазмы в непосредственной близости от диверторных пластин является весьма актуальной. Такие измерения также полезны для исследования режимов с отрывом плазмы от пластин (plasma detachment), оценки интенсивности рециклинга и распыления материала первой стенки [2].

Глобус-М является компактным сферическим токамаком с открытым дивертором, в котором существует возможность получать сильно вытянутую в вертикальном направлении плазму. Плазма может быть ограничена как диафрагмой, так и магнитной сепаратрисой. Система обмоток полоидального магнитного поля позволяет создавать конфигурацию как с верхним, так и с нижним положением X-точки а также с двумя Х-точками.

Ленгмюровские зонды широко используются в диагностике плазмы для локального измерения температуры и концентрации электронов, потенциала плазмы. Если зонд встроен в дивертор токамака, он позволяет также определить плотность потоков тепла и частиц на диверторные пластины. Соответственно с помощью массива таких зондов можно найти пространственные распределения параметров плазмы и сделать оценку полного потока тепла на дивертор.

\section{1. Экспериментальная установка}

На токамаке Глобус-М на нижние диверторные пластины установлен набор из десяти ленгмюровских зондов. Зонды были изготовлены из той же марки графита, что и пластины, и имеют плоскую рабочую поверхность, расстояние между ними в среднем равно $2 \mathrm{~cm}$. Диаметр зондов составляет $8 \mathrm{~mm}$, зазор между пластиной и зондом $0.5 \mathrm{~mm}$, изоляция от диверторных пластин осуществляется с помощью керамических колец (рис. 1). Девять зондов располагались в одном полоидальном сечении, зонд \#10 был смещен в тороидальном направлении на $3 \mathrm{~cm}$ и имел такую же координату по большому радиусу, что и зонд \#6. Большая часть зондов (\#4-10) установлена в районе выхода внешней ветви сепаратрисы на дивертор, так как в сферических токамаках именно в эту область попадают основные потоки тепла и частиц [3]. Плоская форма зондов (собирающая поверхность зонда находится в одной плоскости с поверхностью диверторной пластины) имеет следующие преимущества: тепловая нагрузка на зонд в этом случае минимальна; такие зонды не вносят дополнительного возмущения в плазму и могут служить мониторами тока, текущего на диверторные пластины [4]. Для данного массива зондов была разработана система регистрации, которая могла работать в трех режимах: измерение ионного тока насыщения, измерение плавающего потенциала зондов и запись зондовой вольт-амперной характеристики (BAX). Так как камера токамака изолирована от земли, должна быть изолирована и большая часть зондовой схемы. Это условие было выполнено с использованием блоков АЦП с батарейным питанием 
и оптоволоконной передачей оцифрованных данных в промежутках между разрядами. Частота дискретизации данного АЦП составляет $10 \mathrm{MHz}$, разрядность - $12 \mathrm{bit}$, глубина буфера данных - 1048576 отсчетов. Блок-схема одного из каналов системы регистрации приведена на рис. 2. Питание выходных каскадов схемы, которые обеспечивали подачу напряжения на зонд, осуществляется с помощью конденсаторов достаточно большой емкости С1 и С2, которые заряжаются между разрядами токамака и отсоединяются от блоков питания во время разрядного импульса с помощью электронных ключей. При снятии BAX на зонд подавалось напряжение треугольной формы частотой около $1400 \mathrm{~Hz}$, изменяющееся в диапазоне от $-90 \mathrm{~V}$ до $+50 \mathrm{~V}$, и усреднение, как правило, проводилось за $1-5 \mathrm{~ms}$.

\section{2. Измерение временных зависимостей ионного тока насыщения и плавающего потенциала}

Для апробации диагностики эксперименты проводились в разрядах с омическим нагревом при тороидальном магнитном поле $0.4 \mathrm{~T}$ в диверторной конфигурации с нижней $\mathrm{X}$ точкой. Для встроенных в дивертор ленгмюровских зондов ионный ток насыщения представляет собой поток ионов на пластину, умноженный на элементарный заряд [5]. На рис. 3 приведены временные зависимости плотности ионного тока насыщения для четырех зондов, находящихся вблизи точки пересечения внешней ветви сепаратрисы с диверторной пластиной,

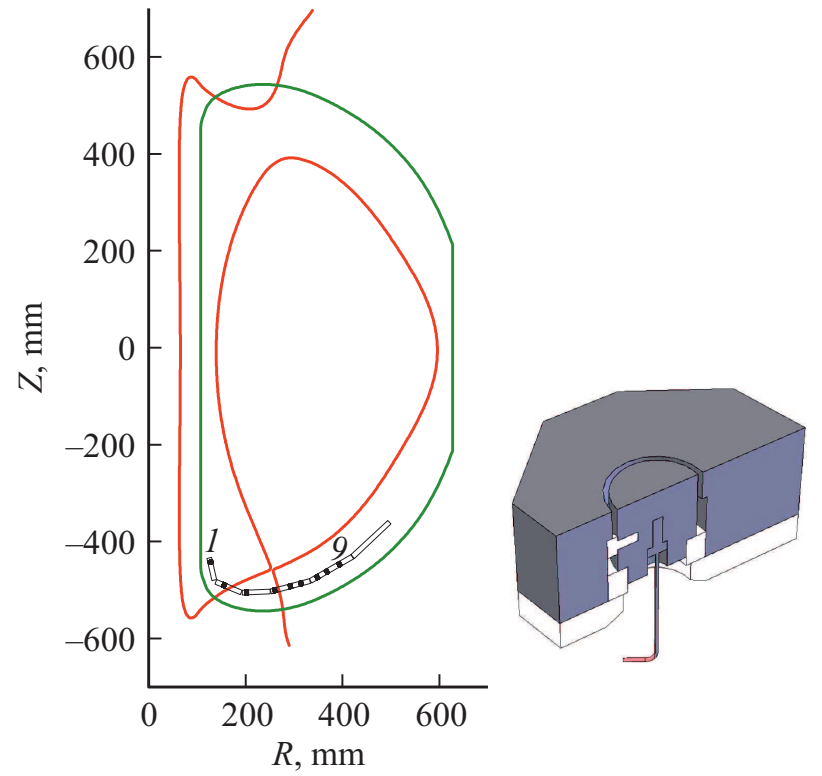

Рис. 1. Расположение встроенных в диверторные пластины ленгмюровских зондов и конструкция зонда. Зонд \#10 имеет ту же координату по большому радиусу, что и зонд \#6. Справа сечение диверторной пластины в плоскости расположения зондов.

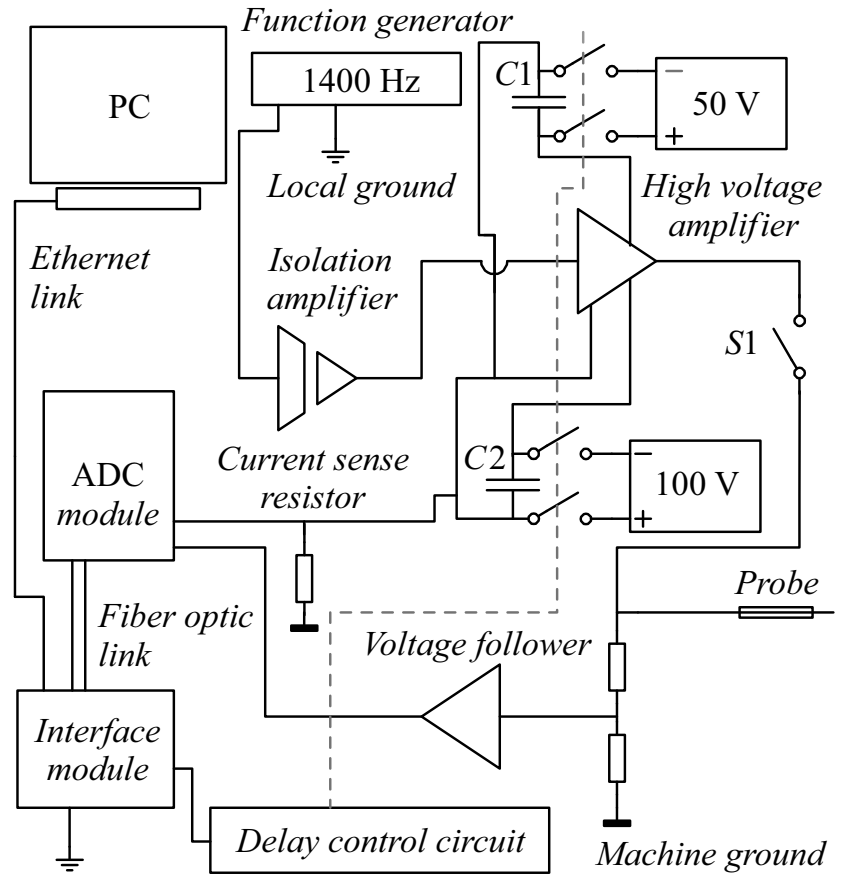

Рис. 2. Блок-схема одного из каналов регистрации. Используются блоки АЦП с батарейным питанием, земля которых присоединена к корпусу токамака. Переключатель $S 1$ используется для перевода схемы в режим измерения плавающего потенциала.

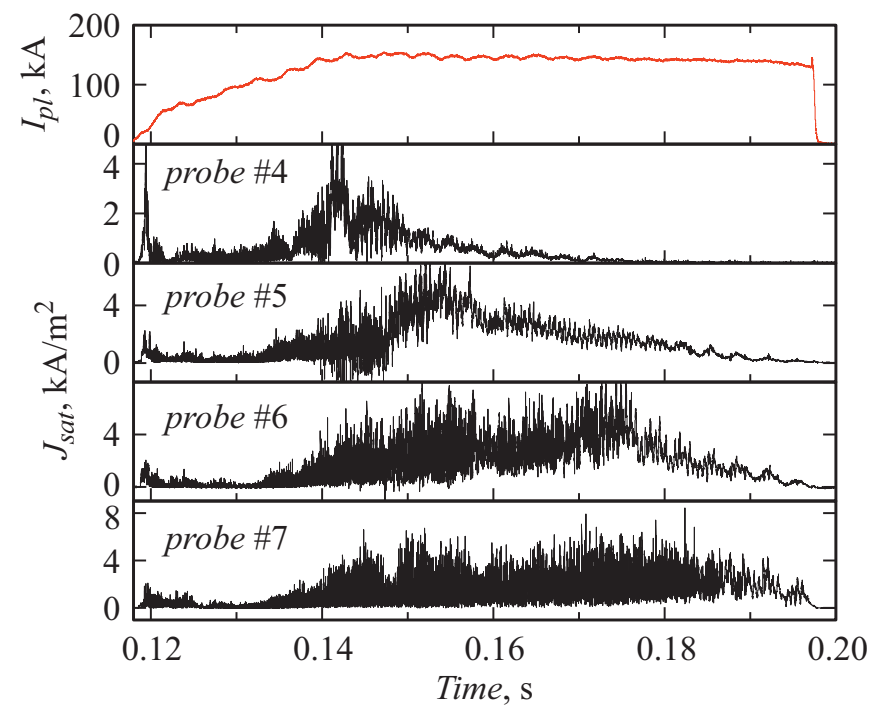

Рис. 3. Временные зависимости плотности ионного тока насыщения для диверторных зондов, находящихся в районе внешней ветви сепаратрисы. Разряд \#34353, ток плазмы $150 \mathrm{kA}$, среднехордовая плотность $\bar{n}_{e}=2.6 \cdot 10^{19} \mathrm{~m}^{-3}$. Номера зондов соответствуют рис. 1 .

т.е. в области, где плотность потока тепла максимальна (strike-point, SP). Номера зондов соответствуют рис. 1. Можно заметить, что, начиная с некоторого момента времени, величина флуктуаций тока значи- 

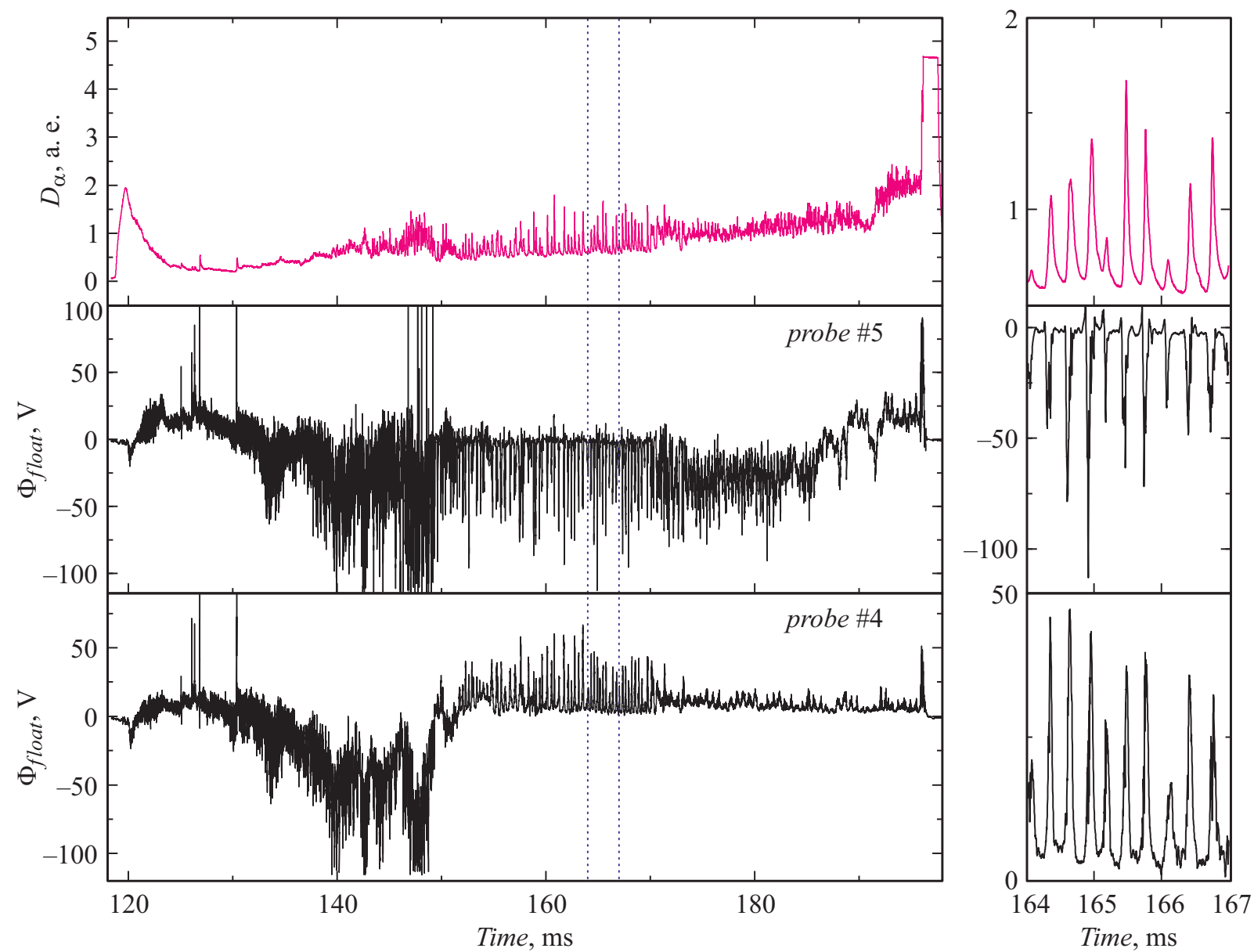

Рис. 4. Влияние периферийной неустойчивости (ELM) на плавающий потенциал диверторных зондов, находящихся вблизи внешней ветви сепаратрисы в SOL (зонд 5) и PFR (зонд 4). Также приведен сигнал, пропорциональный интенсивности линии D-alpha. Область, выделенная пунктирными линиями, показана справа в увеличенном масштабе. B момент ELM возрастание электронной температуры приводит к резкому разнонаправленному изменению плавающих потенциалов.

тельно уменьшается, причем чем больше радиальная координата зонда, тем позже наступает этот момент (от $150 \mathrm{~ms}$ для зонда \#4 до $185 \mathrm{~ms}$ для зонда \#7). Это происходит потому, что в токамаке Глобус-М в течение разряда токамака SP движется наружу со скоростью приблизительно $1 \mathrm{~m} / \mathrm{s}$, и зонды последовательно попадают в область под X-точкой, ограниченной внутренней и внешней ветвями сепаратрисы (private flux region, PFR). Данное значение скорости подтверждается реконструкцией магнитной конфигурации по коду EFIT и записями инфракрасной видеокамеры [6]. Очень похожее поведение сигналов диверторных зондов наблюдалось на сферическом токамаке MAST [7]. Согласно этой работе, низкий уровень флуктуаций в PFR, в частности, можно объяснить тем, что частицы попадают сюда из района около X-точки, где полоидальное магнитное поле близко к нулю. Образующийся шир затрудняет развитие неустойчивостей. Кратковременные отрицательные выбросы на осциллограммах на рис. 3 появляются из-за недостаточного отрицательного напряжения смещения, т. е. в эти моменты времени зонд оказывается под более высоким потенциалом, чем плавающий, и электронный ток становится больше ионного.

На рис. 4 изображены временные зависимости плавающего потенциала в разряде \#35343 для зонда \#5, находящегося вблизи внешней ветви сепаратрисы в SOL (Scrape-of-Layer, обдирочный слой плазмы) и для зонда \#4, который находится в PFR. Также приведен сигнал, пропорциональный интенсивности линии D-alpha. Ток плазмы составлял $200 \mathrm{kA}$, среднехордовая плотность $3.4 \cdot 10^{19} \mathrm{~m}^{-3}$. Приблизительно на $149 \mathrm{~ms}$ происходит переход в режим с улучшенным удержанием (Н-моду), при этом развиваются МГД неустойчивости, локализованные на границе плазмы (edge localized mode - ELM). Во время таких событий за время порядка $100 \mu \mathrm{s}$ происходит выброс частиц в пристеночную плазму, пиковая тепловая нагрузка на диверторные пластины значительно возрастает [2,8], а на сигналах датчиков D-alpha появляются характерные пики. На сигналах плавающих потенциалов, приведенных на рис. 4, также легко видеть четкие пики с амплитудой до $100 \mathrm{~V}$, синхронизованные с пиками на сигнале D-alpha. Причем на зонде, распо- 


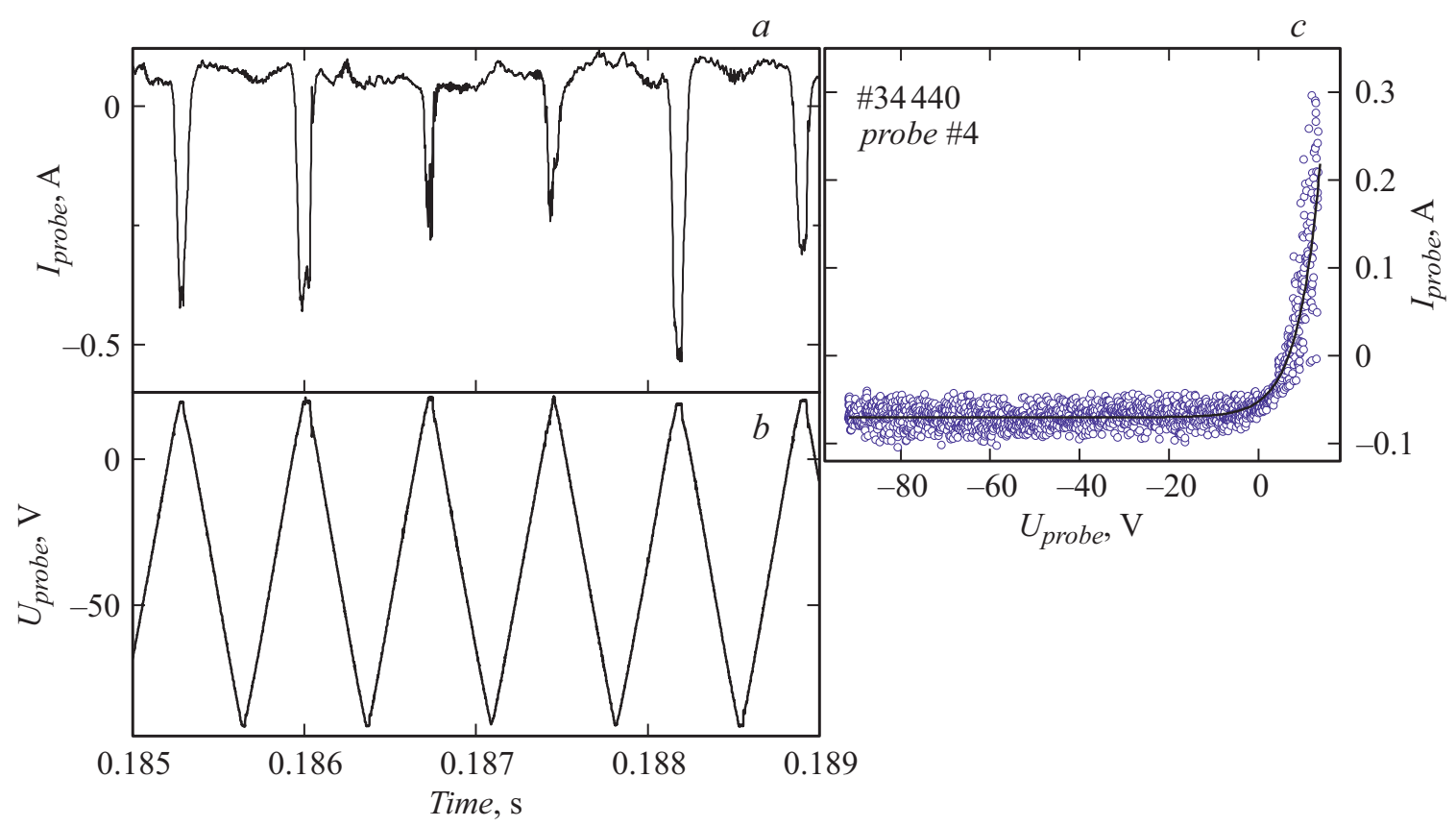

Рис. 5. $a, b-$ осциллограммы зондового тока и напряжения на зонде соответственно; $c$ - кружками обозначены эти данные в виде BAX (здесь положительным считается электронный ток), сплошная линия показывает аппроксимацию для нахождения электронной температуры, ионного тока насыщения и плавающего потенциала.

ложенном в SOL (\#5), наблюдается резкое уменьшение плавающего потенциала, а на зонде в PFR (\#4) наоборот, резкое повышение. По-видимому, такие изменения могут быть связаны с существенным увеличением электронной температуры вблизи диверторных пластин. Подобные явления детально исследовались в работе [5], где также наблюдались разнополярные пики на сигналах диверторных зондов, и где было высказано предположение, что в условиях развития ELM в SOL могут протекать импульсные токи, которые имеют в основном термоэлектрическую природу.

\section{3. Измерение профилей параметров плазмы вблизи диверторных пластин}

Согласно [9], ВАХ зонда можно описать формулой

$$
I_{p r}=I_{\text {isat }}\left[1-\exp \left\{e\left(V_{p r}-V_{f l}\right) / k T_{e}\right\}\right]
$$

где $I_{p r}$ и $V_{p r}$ - ток зонда и напряжение на нем, $I_{i s a t}-$ ионный ток насыщения, $T_{e}$ - температура электронов, $V_{f l}$ - плавающий потенциал. Для нахождения этих трех параметров экспериментально записанная BAX аппроксимировалась зависимостью (1) с помощью стандартного математического пакета. Для обработки, как правило, выбирались разряды с низкой МГД активностью, и использовался участок ВАХ, где ток зонда по абсолютной величине не превосходит $I_{\text {isat }}[3,10]$. Типичные осциллограммы напряжения и тока зонда при записи $\mathrm{BAX}$, а также результат аппроксимации, приведены на рис. 5. Следует отметить, что, несмотря на плоскую форму зондов, в нашем случае, в отличие, например, от [11], довольно четко регистрируется ионный ток насыщения, что может быть связано с тем, что угол между поверхностью зонда и силовой линией магнитного поля превышает $5^{\circ}$ для большинства исследуемых разрядов [12]. Измерив температуру электронов вблизи дивертора и ионный ток насыщения, можно оценить такой важный для токамака параметр, как плотность потока тепла на диверторные пластины. Как известно, каждая ион-электронная пара, проходящая через слой объемного заряда на стенку токамака, переносит энергию из плазмы, которую характеризует суммарный коэффициент передачи тепла в слое $\gamma$, теоретическое значение которого определяется выражением [2]:

$$
\begin{aligned}
\gamma= & 2.5 \frac{T_{i}}{T_{e}}+\frac{2}{1-\delta_{e}} \\
& -0.5 \ln \left[\left(2 \pi \frac{m_{e}}{m_{i}}\right)\left(1+\frac{T_{i}}{T_{e}}\right)\left(1-\delta_{e}\right)^{-2}\right] .
\end{aligned}
$$

Здесь $T_{i}$ - температура ионов, $\delta_{e}-$ коэффициент вторичной электронной эмиссии обращенной к плазме поверхности. Обычно делают предположение, что $T_{i}$ не сильно отличается от $T_{e}$, что дает значение $\gamma=7$. Соответственно для используемых зондов плотность потока тепла на диверторные пластины можно найти по формуле $q=\gamma k T_{e} I_{i s a t} /\left(e S_{p r}\right)$, где $S_{p r}$ - площадь собирающей поверхности зонда. В условиях описываемых экспериментов движение SP вдоль зондов в течение разряда (рис. 4) в некоторых случаях позволяло улучшить 

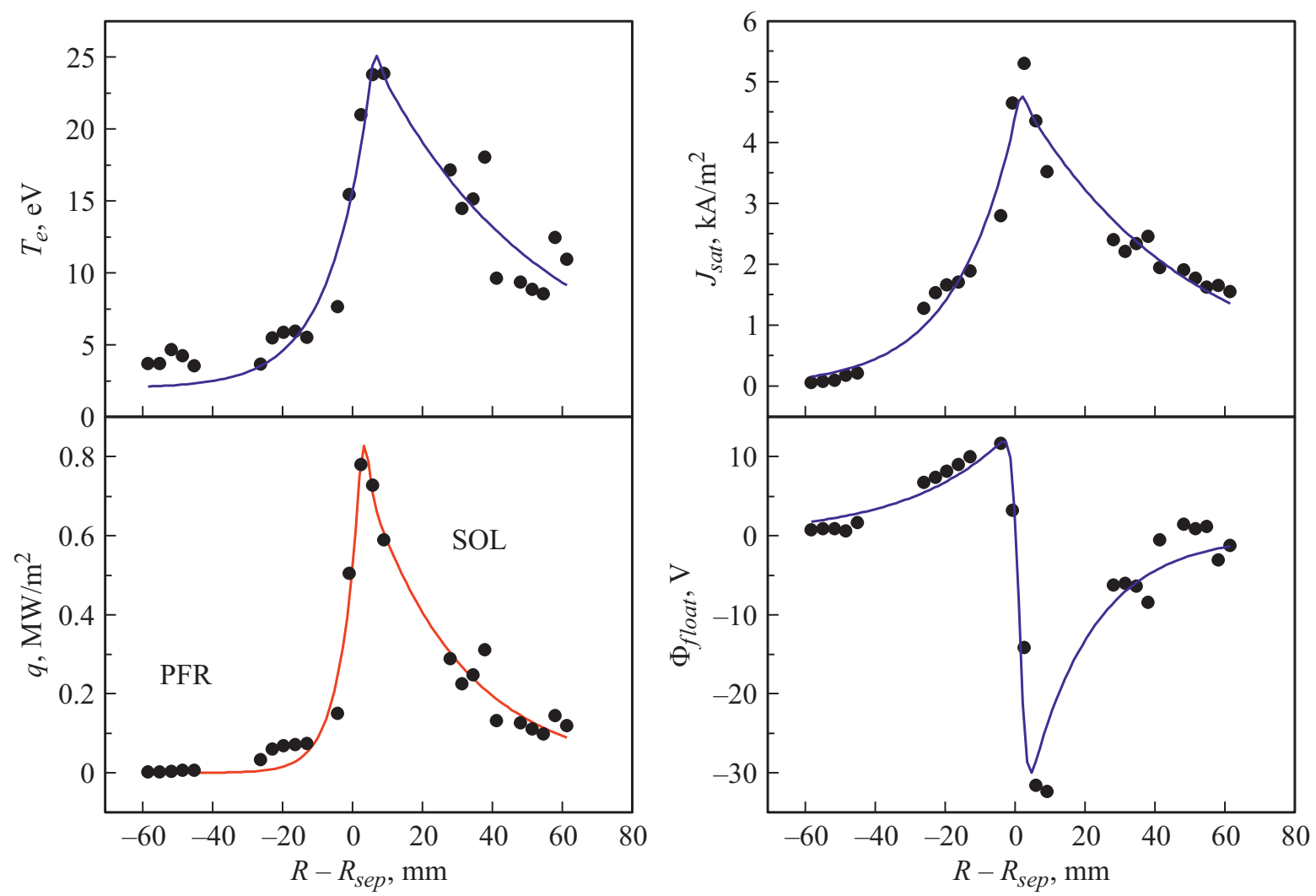

Рис. 6. Профили плотности ионного тока насыщения, температуры электронов, плотности теплового потока и плавающего потенциала вблизи точки выхода сепаратрисы на внешнюю диверторную пластину. Использовалось пространственное сканирование в течение $12 \mathrm{~ms}$ при движении SP наружу со скоростью около $1 \mathrm{~m} / \mathrm{s}$. Время усреднения одной точки $3 \mathrm{~ms}$.

пространственное разрешение описываемой диагностики по сравнению с более ранними измерениями [13]. Если на рассматриваемом интервале времени параметры плазмы меняются несущественно, то можно совместить на одном графике профили, записанные в разные моменты времени, сдвинув их вдоль большого радиуса с учетом скорости движения SP [7]. Использование данного метода проиллюстрировано на рис. 6, где приведены пространственные распределения температуры электронов, плотности ионного тока насыщения, плотности потока тепла и плавающего потенциала для разряда \#34354. Ток плазмы составлял $150 \mathrm{kA}$, среднехордовая плотность $3 \cdot 10^{19} \mathrm{~m}^{-3}$. Как и следовало ожидать, более низкий уровень флуктуаций в PFR приводит к меньшему разбросу точек относительно усредненного профиля. Характерный масштаб спада плотности потока тепла вдоль пластины может быть оценен как $\lambda_{q t} \approx 25 \mathrm{~mm}$, что с учетом расширения полоидального магнитного потока в пограничном слое соответствует скейлингу [14]. Положение максимумов на профилях удовлетворительно согласуется с результатами реконструкции магнитной конфигурации по коду EFIT, некоторый сдвиг наружу может быть связан с диффузией в область PFR [15].

На основе приведенных измерений плавающего потенциала зонда и электронной температуры можно найти потенциал плазмы относительно камеры вблизи диверторной пластины. Согласно [2], для дейтериевой плазмы $V_{p l}=V_{f l}+3 k T_{e} / e$. С использованием этого соотношения на рис. 7 построен профиль отношения потенциала плазмы к температуре электронов $e V_{p l} / k T_{e}$. Отличие этого отношения от 3 (если считать потенциал камеры равным нулю) свидетельствует о появлении тока из плазмы на пластину. Это подтверждает показанный на этом же рисунке профиль плотности зондового тока при нулевом смещении на нем, т.е. зонды выступают в роли мониторов тока. Данные значения тока были взяты из тех же зондовых ВАХ, которые использовались для построения профилей на рис. 6. Как и следовало ожидать из (1), если потенциал плазмы становится меньше $3 k T_{e} / e$, то электронный ток на пластину резко (экспоненциально) возрастает. Если же потенциал плазмы превышает $3 k T_{e} / e$, то ток ионов становится больше тока электронов, но при этом его зависимость от $e V_{p l} / k T_{e}$ более слабая. Следует отметить, что в формуле (2) предполагается, что ток ионов равняется току электронов и при этом значение $\gamma$ близко к минимальному [2]. То есть в условиях разряда \#34354 наличие значительного электронного тока на пластину вблизи SP, по-видимому, приводит к существенному занижению плотности теплового потока, вычисляемой согласно (2). 


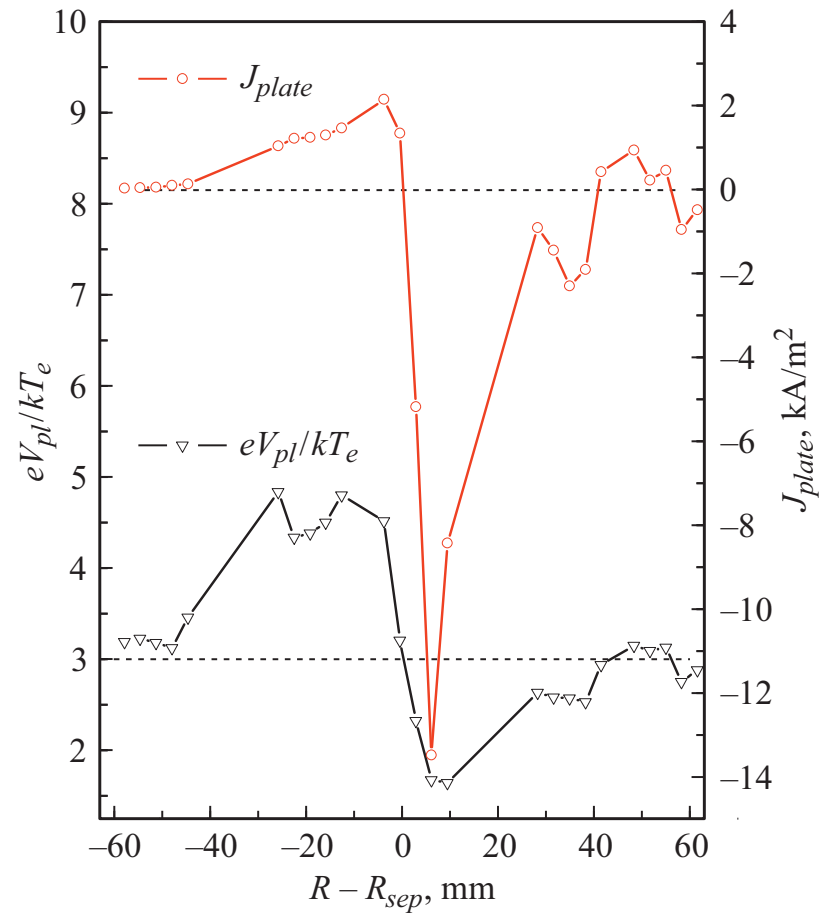

Рис. 7. Профили плотности тока на внешнюю диверторную пластину и отношения потенциала плазмы к электронной температуре для разряда \#34354.

Параметры краевой плазмы токамака Глобус-М для схожих разрядов моделировались кодом B2SOLPS5.2 с учетом всех дрейфов и токов [13]. Плотность тока на пластину, полученная в моделировании, имеет те же особенности, что и измеренная экспериментально - в области PFR ток направлен на пластину и его плотность меньше плотности ионного тока насыщения. Снаружи от SP ток меняет знак и течет с пластины, совпадая по направлению $\mathrm{c}$ термотоком, текущим с более горячей наружной пластины на более холодную внутреннюю. Величина плотности тока с пластины превышает плотность ионного тока насыщения в узкой области SOL вблизи SP.

\section{Заключение}

Таким образом, с помощью массива ленгмюровских зондов, встроенных в дивертор токамака Глобус-М, для разрядов с омическим нагревом определены основные параметры плазмы вблизи выхода внешней ветви сепаратрисы на диверторные пластины. При развитии неустойчивостей ELM зафиксированы импульсы плавающего потенциала зондов с разной полярностью, которые коррелируют с сигналами датчиков D-alpha. Измерена плотность электрического тока на диверторную пластину. Внутри SOL вблизи SP ток течет с наружной пластины и приблизительно в два раза превышает ионный ток насыщения. Неучет тока с пластины приводит к занижению оценки плотности потока тепла, полученной из результатов зондовых измерений. В дальнейшем планируется увеличить число зондов, установив их также и на верхний купол токамака.

\section{Финансирование работы}

Разработка массива диверторных ленгмюровских зондов для исследований пристеночной плазмы выполнена в рамках государственного задания Министерства науки и высшего образования Российской Федерации. Эксперименты проводились на уникальной научной установке „Сферический токамак Глобус-М““, входящей в состав федерального Центра коллективного пользования ФТИ им. Иоффе „Материаловедение и диагностика в передовых технологиях““ (уникальный идентификатор проекта RFMEFI62119X0021). Численное моделирование проводилось в рамках проекта Министерства науки и высшего образования Российской Федерации № 0784-2020-0020.

\section{Конфликт интересов}

Авторы заявляют, что у них нет конфликта интересов.

\section{Список литературы}

[1] G.F. Counsell, A. Kirk, J.-W. Ahn, A. Tabasso, Y. Yang. Plasma Phys. Control. Fusion, 44, 827 (2002). DOI: 10.1088/07413335/44/6/314

[2] P.C. Stangeby. The Plasma Boundary of Magnetic Fusion Devices (IoP Publishing, Bristol, 2000)

[3] G.F. Counsell, J.-W. Ahn, R. Akers, E. Arends, S.J. Fielding, P. Helander, A. Kirk, H. Meyer, A. Tabasso, H. Wilson, Y. Yang. J. Nucl. Mater., 313-316, 804 (2003). DOI: $10.1016 / \mathrm{S} 0022-3115(02) 01439-3$

[4] J.P. Gunn, C. Boucher, D. Desroches, A. Robert. Rev. Sci. Instrum., 68, 404 (1997). DOI: 10.1063/1.1147840

[5] R.A. Pitts, S. Alberti, P. Blanchard, J. Horacek, H. Reimerdes, P.C. Stangeby. Nucl. Fusion, 43, 1145 (2003). DOI: $10.1088 / 0029-5515 / 43 / 10 / 017$

[6] Н.В. Сахаров, А.В. Воронин, В.К. Гусев, А.А. Кавин, С.Н. Каменщиков, К.М. Лобанов, В.Б. Минаев, А.Н. Новохацкий, М.И. Патров, Ю.В. Петров, П.Б. Щеголев. Физика плазмы, 41, 1076 (2015). DOI: $10.7868 / \mathrm{S} 0367292115120124 \quad$ [N.V. Sakharov, A.V. Voronin, V.K. Gusev, A.A. Kavin, S.N. Kamenshchikov, K.M. Lobanov, V.B. Minaev, A.N. Novokhatsky, M.I. Patrov, Yu.V. Petrov, P.B. Shchegolev. Plasma Physics Reports, 41, 997 (2015). DOI: 10.1134/S1063780X15120120]

[7] G.Y. Antar, G. Counsell, J.-W. Ahn, Y. Yang, M. Price, A. Tabasso, A. Kirk. Phys. Plasmas, 12, 032506 (2005). DOI: $10.1063 / 1.1861894$

[8] A. Kirk, G.F. Counsell, H.R. Wilson, J.-W. Ahn, R. Akers, E.R. Arends, J. Dowling, R. Martin, H. Meyer, M. Hole, M. Price, P.B. Snyder, D. Taylor, M.J. Walsh, Y. Yang. Plasma Phys. Control. Fusion, 46, 551 (2004). DOI: $10.1088 / 0741-3335 / 46 / 3 / 009$

[9] P.C. Stangeby, G.M. McCracken. Nucl. Fusion, 30, 1225 (1990). DOI: 10.1088/0029-5515/30/7/005 
[10] N. Asakura, K. Shimizu, N. Hosogane, K. Itami, S. Tsuji, M. Shimada. Nuclear Fusion, 35, 381 (1995). DOI: 10.1088/0029-5515/35/4/I02

[11] J.P. Gunn, C. Boucher, B.L. Stansfield, S. Savoie. Rev. Sci. Instrum., 66, 154 (1995). DOI: 10.1063/1.1145249

[12] A.Q. Kuang, D. Brunner, B. LaBombard, R. Leccacorvi, R. Vieira. Rev. Sci. Instrum., 89, 043512 (2018). DOI: $10.1063 / 1.5023905$

[13] E. Vekshina, I. Senichenkov, V. Rozhansky, E. Kaveeva, N. Khromov, G. Kurskiev, M. Patrov. Plasma Phys. Control. Fusion, 58, 085007 (2016). DOI: $10.1088 / 0741-3335 / 58 / 8 / 085007$

[14] T. Eich, A.W. Leonard, R.A. Pitts, W. Fundamenski, R.J. Goldston, T.K. Gray, A. Herrmann, A. Kirk, A. Kallenbach, O. Kardaun, A.S. Kukushkin, B. LaBombard, R. Maingi, M.A. Makowski, A. Scarabosio, B. Sieglin, J. Terry, A. Thornton. Nucl. Fusion, 53, 093031 (2013). DOI: $10.1088 / 0029-5515 / 53 / 9 / 093031$

[15] A. Gallo, N. Fedorczak, S. Elmore, R. Maurizio, H. Reimerdes, C. Theiler, C.K. Tsui, J.A. Boedo, M. Faitsch, H. Bufferand, G. Ciraolo, D. Galassi, P. Ghendrih, M. Valentinuzzi, P. Tamain. Plasma Phys. Control. Fusion, 60, 014007 (2018). DOI: 10.1088/1361-6587/aa857b 